\title{
Anabases
}

ANABASES Traditions et réceptions de l'Antiquité

\section{Introduction : Le passé antérieur. Pour une étude de la « Préhistoire » des Anciens}

\section{Pierre Cordier}

\section{(2) OpenEdition}

1 Journals

Édition électronique

URL : http://journals.openedition.org/anabases/2703

DOI : 10.4000/anabases. 2703

ISSN : 2256-9421

Éditeur

E.R.A.S.M.E.

\section{Édition imprimée}

Date de publication : 1 mars 2006

Pagination : 101-109

ISSN : 1774-4296

\section{Référence électronique}

Pierre Cordier, «Introduction : Le passé antérieur. Pour une étude de la « Préhistoire » des Anciens »,

Anabases [En ligne], 3 | 2006, mis en ligne le 01 novembre 2011, consulté le 20 octobre 2019. URL:

http://journals.openedition.org/anabases/2703; DOI : 10.4000/anabases.2703 
Anabases 3 (2006), p. 101-109

\section{Introduction : Le passé antérieur. Pour une étude de la «Préhistoire" des Anciens}

PierRe Cordier

Si elle n’est pas utilisée dans un sens affadi, mais pour évoquer bel et bien des conceptions des temps originels, l'expression de "Préhistoire des Anciens» doit heurter l'oreille de l'historien, de l'anthropologue, du philologue et de tout spécialiste des mondes anciens. Le mot de préhistoire, création du XIXe siècle ${ }^{1}$, est en effet inconnu dans l'Antiquité ; l'idée n'y semble a priori pas repérable. L'anachronisme se double d'un hiatus épistémologique : le mot et la notion ont même été constitués, dans une large mesure, pour traduire une méthode et des objets bien distincts de ceux que se proposaient les Antiquaires et pour réaliser la convergence des sciences de la nature et

1 Le mot apparaît en anglais en 1851 chez Daniel Wilson (Prehistoric Man : Researches into the Origin of Civilisation in the Old and the New World, Londres), mais est popularisé par John Lubbock en 1865 (Prehistoric Times, Londres) ; dès 1874, il entre dans le lexique français, où il supplante un vocable plus ancien, " antéhistorique ", attesté dès 1828 : cf. A. REY (dir.), Dictionnaire historique de la langue française, Paris, Dictionnaires Le Robert, 1993, s. v. "historique", p. 965 et Trésor de la langue française. Dictionnaire de la langue du XIXe et du XXe siècle (1789-1960), Paris, Gallimard s. v. "préhistoire", p. 1050 (la datation donnée pour la première occurrence du terme anglais est fausse). Pour une histoire de la question, on se reportera à N. RICHARD, La préhistoire en France dans la second moitié du XIXe siècle (1859-1904), Thèse de doctorat, université de Paris I, 1992 ; Id. (intr., trad., comm.), Linvention de la préhistoire : anthologie, Paris, presses Pocket, 1992 ; W. STOCZKOWSKI, "La préhistoire : les origines du concept", N. Richard (dir.), Histoire de la préhistoire: Bulletin de la Société préhistorique française $\mathrm{n}^{\circ}$ spécial 90.1-2 (1993), p. 13-21 ; D.R. Kelley, "The Rise of Prehistory”, Journal of World History 14.1 (2003), 40 paragraphes, consulté en novembre 2005

<http ://www.historycooperative.org/journals/jwh/14.1/kelley.html>. 
des sciences de l'Homme ${ }^{2}$; pour autant les études préhistoriques, à mesure que s'affirmait leur autonomie disciplinaire et méthodologique, ont longtemps été traitées par l'Histoire académique pour un rameau dissident et corrompu de l'étude des Antiquités 3 .

L'historiographie de la notion de préhistoire fait pourtant apparaitre, contrairement à une idée reçue tenace, l'absence de démarcation franche entre les représentations de l'humanité antérieures au XVII e siècle et celles que développent le XIXe et le $\mathrm{XX}$ siècles, en dépit de nouvelles méthodes d'investigation et des nouvelles données matérielles disponibles. L'idée d'une "invention de la préhistoire " au XIXe siècle est à relativiser, d'abord parce qu'elle procède, en grande partie, du discours d'autocélébration qui tend à accompagner la constitution de toute nouvelle discipline intellectuelle et de la promotion intensive, par les préhistoriens, de la nouveauté et du caractère révolutionnaire de leurs trouvailles. De fait, la remise en cause du schéma biblique des origines humaines ou de leurs modèles philosophiques hérités de l'Antiquité remonte au moins au début de l'époque moderne, sinon plus haut ${ }^{4}$. La persistance des idées reçues est un second facteur de cette absence de démarcation : comme l'a montré Stoczkowski, l'" anthropologie savante " reprend le plus souvent à son compte, hier comme aujourd'hui, les catégories de l'« anthropologie naïve 5 ». Ainsi, lorsqu'il se pose de nos jours la question des jalons et des critères de l'hominisation, le préhistorien reprend-il à son compte des schémas classiques, longtemps débattus non seulement dans le cadre omniprésent du schéma biblique de la Création, mais aussi par référence

2 Cf. M. Foucault, L'archéologie du savoir, Paris, Gallimard, 1975 ; A. SCHNAPP, La Conquête du passé. Aux origines de l'archéologie, Paris, Éditions Carré (LGF), 1993, notamment p. 267-286, 335-387 ; ci-après, les remarques introductives de M.-L. DesClOS, "Représentations platoniciennes du temps d'avant". Cf. aussi C. BlanCKAERT, "Une anthropologie de transition. Lacépède et l'histoire naturelle de l'homme", Annales Benjamin Constant 13 (1992), p. 95-111 ; J.-Y. PAUTRAT, "L'homme primitif, la vie, L'histoire" in A. et J. Ducros (éd.), L’homme préhistorique. Images et imaginaire, Paris, L'Harmattan, 2000, p. 139-157, notamment p. 141-142 sur la situation de l'Homme, chez Boucher de Perthes, dans "le grand arbre de la vie".

Cf. N. RiCHARD, "L'homme invisible. Les historiens français et l'homme fossile à la fin du XIXe siècle”, in A. et J. DuCros (éd.), L’homme préhistorique... cit., p. 63-79, qui souligne le rôle de Camille Jullian et de son "Plaidoyer pour la préhistoire", in Au seuil de notre histoire, I, Paris, Boivin, 1930, p. 52-74. Prehistory, Edimbourg, Edinburgh University Press, 1988 ; J. MALINA et Z. VAsiceK, Archaeology Yesterday and Today : The Development of Archaeology in the Sciences and Humanities, Cambridge, Cambridge University Press, 1990 et A. SCHNAPr, La Conquête du passé.

W. STOcZKowski, Anthropologie naïve, anthropologie savante. De l'origine de l'homme, de l'imagination et des idées reçues (Empreintes de l'Homme), Paris, CNRS éditions, 1994 ; cf. aussi C. COHEN, L'homme des origines. Savoirs et fictions en préhistoire, Paris, Seuil, 1999. 
expresse à la tradition grecque et romaine antique ${ }^{6}$. Les caractères réputés distinctifs de l'humanité (bipédie, langage, habileté à produire et utiliser des outils) ou le rôle de la nécessité et/ou du besoin dans l'évolution des hominidés illustrent bien cette tendance. Un exemple suffira à l'attester : chacun connaît l'idée, vulgarisée depuis les années 1980 par une abondante littérature et, tout récemment, par des émissions télévisées européennes, que l'hominisation résulterait, à son origine, d'une rupture écologique - l'abandon de la jungle et le peuplement des savanes africaines - et de l'adaptation d'un être naturellement mal armé aux contraintes d'un environnement difficile 7 .

Quoique inspirée par l'évolutionnisme darwinien, qui voit dans l'utilité pour la survie le critère discriminant des mutations biologiques, cette conception ne se différencie guère, dans ses principes, de certains mythes antiques qui dramatisent le passage d'un environnement facile et d'une condition quasi animale aux affres d'un monde où la nécessité de l'effort est le principe de l'anthropogenèse ${ }^{8}$. De même que la fin de l'âge d'or chez Virgile et Ovide confronte l'homme au manque et constitue le point zéro de l'histoire proprement humaine, de même, en chassant Adam et Ève du jardin d'Éden, le dieu de la Genèse énonce le programme de la nouvelle condition humaine : pénurie, travail et mortalité. "Parce que tu as cédé à la voix de ton épouse, et que tu as mangé de l'arbre dont je t'avais enjoint de ne pas manger, maudite est la terre à cause de toi ; c'est avec peine que tu en tireras ta nourriture, tant que tu vivras. Elle produira pour toi des ronces et des épines, et tu mangeras de l'herbe des champs. C'est à la sueur de ton visage que tu mangeras du pain - jusqu'à ce que tu retournes à la terre d'où tu as été tiré : car poussière tu fus, et à la poussière tu retourneras " $G n$ III, 17-19).

Il y a un point commun entre le Dieu sévère de la Torah et l'évolutionnisme : ils font de l'homme actuel le fils de la nécessité - au sens de contrainte environnementale -, un être condamné à s'engendrer lui-même dans la douleur ; l'hominisation est, en ce sens, le travail d'un interminable accouchement. Cette convergence n'est pas un simple effet de surface ${ }^{9}$. Dans les trente dernières années, l'anthropologie des sociétés paléolithiques a remis en cause de manière radicale ce type de schéma, en dressant contre l'idée de nécessité celle de besoin endogène. Citons, par exemple, les études de Marshall Sahlins, qui décrivent parfois les sociétés de chasseurs-cueilleurs comme des univers d'abondance et des milieux dans lesquels le travail n'est qu'une occupation minimale : l'apparition des marqueurs culturels de l'humanité (vêtement, outillage, etc.) - voire, à leur suite, certaines évolutions physiologiques qui en découlent -, ne

6 Cf. W. STOCZKowski, "Origines de l'homme. Quand la science répète le mythe", La recherche 244 (juin 1992), p. 746-752.

7 Cf. dernièrement le film documentaire de J. Malaterre, L'odyssée de l'espèce, coproduction franco-germano-italienne de 90 minutes (janvier 2003).

8 Cf. P. Cordier, Nudités romaines. Un problème d'histoire et d'anthropologie (Études anciennes, série latine, 63) Paris, Les Belles Lettres, 2005, p. 22-50.

9 Cf. W. STOCZKOWSKI, "Origines de l'homme. Quand la science répète le mythe". 
constitueraient pas la réponse à une série de contraintes, de besoins et de manques, mais autant de choix délibérés, issus de considérations non nécessairement - ni principalement - utilitaires 10 .

Le fait de constater la convergence des représentations contemporaines de l'hominisation et des modèles antiques de l'anthropogenèse ne déprécie en rien la réflexion des préhistoriens, bien au contraire : il rend sensible la nature fondamentalement anthropologique de la question de la préhistoire. L'enjeu de cette question n'est autre qu'une définition de l'humain. C'est pourquoi l'anthropogenèse, comme représentation du degré zéro de l'humanité, est toujours abordée sous l'espèce d'un récit à caractère étiologique. Les ressemblances entre les théories contemporaines et antiques sur la préhistoire tiennent probablement davantage à une similitude fonctionnelle - leur place dans le système de représentation d'un fait humain saisi fatalement par induction - qu'à des références directes, même s'il ne faut pas sous-estimer la vigueur des héritages antiques, notamment en matière de questions philosophiques.

L’intérêt pour la "Préhistoire des Anciens " n'est pas nouveau. Pour passer sur les dissertations qui, autour de 1900, abordent la question sous l'angle de la doxographie ${ }^{11}$, il faut citer un ouvrage important, le premier volume d'une longue série, paru en 1935 sur la notion de primitivisme dans l'Antiquité : le recueil de textes antiques, accompagné de volumineux prolégomènes, qu'éditent Arthur $\mathrm{O}$. Lovejoy et George Boas ${ }^{12}$. C'est une entreprise d'histoire des idées, impliquant notamment philologie, sociologie, sociologie et philosophie, en une approche pluridisciplinaire contemporaine, mais distincte de l'école des Annales (et qui reste à étudier en France). L'objet de Lovejoy et Boas est le "primitivisme ": les auteurs en repèrent deux versions, dont les modulations fournissent le plan du livre :

- le primitivisme chronologique, une philosophie de l'histoire qui voit dans un moment particulier du temps ancien le meilleur état du monde ou l'état le plus enviable de la condition humaine. Ce primitivisme connaît de multiples avatars : les théories finitistes bilatérales ou unilatérales (qui prêtent au monde un début et une fin, ou seule-

10 Cf. M. D. SAhlins, Au cour des sociétés. Raison utilitaire et raison culturelle, Paris, Gallimard, 1980 ; Id., Âge de pierre, âge d'abondance : l'économie des sociétés primitives, préf. de Pierre Clastres, Paris, Gallimard, 1976 (1ère éd. Anglaise 1972) ; P. Clastres, "Les marxistes et leur anthropologie", Libre 3 (1978), p. 135-149, repris in Id., Recherches d'anthropologie politique, Paris, Seuil, 1980, p. 157-170 ; Pascal PICQ, Les origines de l'homme, Paris, Seuil, 2005, p. 239-249.

11 Cf. notamment J. WOLTJER, Lucretii philosophia cum fontibus comparata. Specimen litterarum quo inquiritur quatenus Epicuri philosophiam tradiderit Lucretius, Groningen, s. n. (Noordhoff), 1877, réimp. New York, Garland, 1987.

12 A. O. Lovejoy, J. BoAs (éd.), Primitivism and related ideas in Antiquity (Contributions to the history of primitivism, 1), Baltimore, The John Hopkins Press 1935 (prolegomena p. 1-22), réimpr. 1997. 
ment un début ou une fin), les théories ondulatoires (p. ex. pour la succession des empires), les théories du déclin, de l'ascension, des cycles, les théories infinitistes ;

- le primitivisme culturel, expression du mécontentement que la civilisation inspire aux civilisés, que la nature soit vue comme un milieu doux et harmonique ou, à l'opposé, comme une marâtre cruelle.

Malgré sa raideur, la taxinomie de ce Sourcebook présente un intérêt incontestable : elle met en évidence la combinatoire fort complexe des façons de se représenter les origines l'humanité non civilisée. Elle limite la tentation de cloisonner les discours par genre (mythes, histoire, récits philosophiques, descriptions ethnographiques, etc.) pour révéler, sous la diversité de leurs modes d'expression, des représentations communes, des préoccupations transversales.

La plupart des études postérieures au Primitivism de Lovejoy et Boas sont consacrées à la question des origines. Celle-ci ne se confond pas avec le commencement, notamment parce que le discours antique sur les origines est associé, depuis Aristote, à l'éloge 13. Dans les années cinquante, Elias Bickerman consacra un article fondamental à la question des origines gentium, pour souligner le rôle que jouaient dans la représentation de soi les récits des origines, toujours créateurs de liens et de séparations et organisateurs d'un paysage identitaire ${ }^{14}$. Un séminaire trisannuel de l'université de Bordeaux III a repris cette question en mêlant la perspective générale d'Elias Bickerman à des études monographiques et régionales sur le monde antique ${ }^{15}$. Ses divers articles mettent en évidence l'usage identitaire du récit des origines, soit qu'il cautionne un prestige ou des droits particuliers (on songe immédiatement à l'autochtonie athénienne), soit qu'il fonde ou consolide une relation positive dans le présent en alléguant une parenté ancienne (syngeneia). La revue Uranie. Mythes et littératures, publiée par l'université Charles-de-Gaulle (Lille III) consacre un numéro au thème : Mythe et / ou philosophie dans les textes grecs et latins sur les origines de l'humanité ${ }^{16}$. Malgré le titre, il ne s'agit pas de revenir sur les acquis de Lovejoy et Boas, mais de relativiser l'opposition mythos / logos en insistant sur les stratégies d'énonciation mises en œuvre pour aborder la question des origines de l'humanité. Un colloque tenu à Nice en octobre 1995 sur

13 Aristote, Rhétorique (I, 9, 1367b 28-36). Cf. L. BRISSON, "Le Banquet de Platon et la question des commencements de l'humanité", p. 45-54, notamment p. 45.

14 E. J. BiCKERMAN, "Origines gentium", Classical Philology 47 (1952), p. 65-81 ; réimpr. in Id., Religions and Politics in the Hellenistic and Roman Periods, Como, New Press, 1985, pp. 399-417 ; cf. également M. DeTIENNE, L'invention de la mythologie, Paris, Gallimard, 1981, p. 155-189.

15 V. Fromentin et S. GotTEland (éd.), Origines gentium. Séminaire Bordeaux 1996-1997 (Ausonius-publications. Études, 7), Bordeaux, 2001.

16 J. FABRE-SERRIS (éd.), Mythe et / ou philosophie dans les textes grecs et latins sur les origines de l'humanité. Actes des journées d'études des 13 et 14 novembre 1998, Villeneuve-d'Ascq, université Charles de Gaulle - Lille III (Centre de recherche interdisciplinaire "Mythes et littérature"), Uranie 1 (2000). 
Les origines de l'Homme d'après les Anciens réunit diverses études de cas sur des aires de civilisation (Mésopotamie, Extrême Orient) ou sur des corpus spécifiques, en privilégiant deux axes : les éléments du récit des origines et ses modalités narratives ${ }^{17}$. Mentionnons encore le cinquième colloque du PARSA (Pôle Alpin de recherches sur les Sociétés Antiques, compagnon de route de l'équipe ERASME) sur l'« antique des Anciens 18 ». D’une façon plus générale, et pour considérer le problème au sein l'historiographie contemporaine des mondes anciens, la question du rapport que l'Antiquité pouvait entretenir avec sa préhistoire se pose dans le contexte d'un retour de l'histoire sur elle-même, et d'un intérêt récent pour les archéologies antiques, dont témoignent un grand nombre de synthèses récentes ${ }^{19}$.

Plutôt qu'à ses origines, susceptibles de reculer à l'infini, il s'agit ici de s'attacher au commencement de l'humanité, c'est-à-dire à la façon dont l'Antiquité se pose la question de la genèse de l'état social, au passé antérieur selon les Anciens. Nous avons déjà relevé les analogies que les enjeux anthropologiques de ces questions font apparaitre entre les controverses antiques et les débats modernes et contemporains. Dans les termes de la philosophie ou de la sociologie modernes comme pour les historiens, les ethnographes ou les philosophes antiques, la saisie de la "préhistoire » définit en outre son objet, l'Homme, à l'intérieur d'un espace orthonormé, dont nature et culture définissent les axes des abscisses et des ordonnées ${ }^{20}$. Une difficulté supplémentaire s'ajoute à cette situation relative de l'objet humain, pour accentuer traîtreusement l'air de famille qu'ont les interrogations des Anciens et les enquêtes du présent : comme il

17 Les origines de l'homme d'après les anciens. Actes du colloque organisé par le Centre de recherche d'histoire des Idées, les 5-7 octobre 1995 à la Faculté des Lettres de Nice (Publ. De la Faculté des Lettres, Arts et Sciences humaines de Nice, n. s., 46), Nice, Université de Nice Sophia-Antipolis 1998.

18 G. Cajani, D. Lanza, (éd.), L'antico degli Antichi, V Convegno Internazionale del P.A.R.S.A., Pavia 14-15 Ottobre 1999, Palermo, Palumbo (Letteratura classica, 23), 2001.

19 Cf. J. BoARDMAN, The Archaeology of Nostalgia: How the Greeks Re-created their Mythical Past, Londres, Thames \& Hudson, 2001 ; S.E. AlCOCK, Archaeologies of the Greek Past: Landscape, Monuments and Memories, Cambridge, Cambridge University Press, 2002 ; S.E. Alcock, J.F. Cherry, J. Elsner, Pausanias : Travel and Memory in Roman Greece, Oxford, Oxford University Press, 2001.

20 Cf. G. CAmps, s. v. "Préhistoire", in A. Burguière (dir.), Dictionnaire des sciences historiques, Paris, PUF, 1986, p. 537-543, notamment p. 538 : "La préhistoire serait une branche de la philosophie ou bien une annexe de la paléontologie" ; P.J. WATSON, "Archaeology, anthropology, and the culture concept", American Anthropologist 97.4, 1995, p. 683-694 ; W. STOCZKOWSKI, "Les conceptions de la 'nature humaine' dans les scénarios de l'hominisation", in C. CALAME et M. KILANI (dir.), La fabrication de l'humain dans les cultures et en anthropologie, Lausanne, Payot, 1999, p. 127-138. Pour mesurer le caractère traditionnel du débat, cf. également N. RiCHARD, "Entre matérialisme et spiritualisme, les préhistoriens dans la seconde moitié du XXe siècle”, in A. ET J. DUCROS, F. JOUlian (dir.), La culture est-elle naturelle? Histoire, épistémologie et applications récentes $d u$ concept de culture, Paris, Errance, 1998, p. 25-40. 
s'agit, bon gré mal gré, de prendre acte d'un processus de changement - de l'état présocial à l'état de société -, le recours inévitable à l'idée de progrès peut nourrir l'illusion d'une filiation directe ou d'une similitude ${ }^{21}$. Il tend à gommer les considérants propres et les données spécifiques de la réflexion antique sur le passé antérieur, d'autant plus aisément que, depuis une quinzaine d'années, l'historiographie contemporaine tend, par un effet de balancier bien souvent éprouvé, à substituer à une conception souvent inavouée des sociétés antiques comme des structures figées, "froides ", l'image d'organismes dynamiques et " chauds ", intégrant le changement et l'histoire dans leur définition de soi ${ }^{22}$. Mais il faut être attentif à rendre les interrogations antiques à leur contexte, et les Anciens à leur irréductible éloignement. C'est pourquoi le problème est ici abordé de préférence sous l'angle politique.

Dans l'Antiquité grecque et romaine, le politique prend en effet la dimension d'un phénomène social total et affecte toutes les modalités de l'action et de la pensée. À juste titre, la recherche contemporaine s'est attachée à ses expressions spécifiques - la cité ou la citoyenneté -, pour saisir leur développement historique et idéologique, leurs variations et leur extension dans l'espace, leurs mutations et leur continuité dans le temps, voire leurs implications anthropologiques. Le manque d'intérêt, jusqu'à ces dernières années, pour la "préhistoire " des Anciens traduit la prévalence et l'évidence du politique, mais aussi l'idée que mythes et " archéologies " antiques ne vaudraient guère que comme un argumentaire idéologique au service de leurs contemporains. Pourtant, loin de se laisser saisir seulement, dans la pensée du passé, comme phase d'un développement historique ou étape antérieure à l'apparition de l'homme politique, l'état présocial de l'Homme, aux yeux des auteurs Anciens, pouvait s'incarner et s'animer hic et nunc, dans des monuments hérités d'un passé obscur, dans leurs étiologies, voire dans des groupes humains contemporains dont le comportement ne montrait, aux yeux d'un ethnographe grec ou romain, aucun signe distinctif de l'humanité. C'est cette présence actuelle du passé, le vocabulaire grec en témoigne, qui distingue, parmi

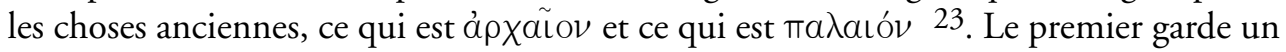

21 Sur la spécificité de l'idée antique de progrès et la doxographie de la notion, on peut se reporter à A. NOVARA, Les idées romaines sur le progrès d'après les écrivains de la République (essai sur le sens latin du progrès) (Publications de la Sorbonne, 1), Paris, Belles Lettres, 1982.

22 Pour ne citer qu'un seul exemple de cette tendance, nous évoquerons le recul de l'expression de "Bas Empire " et la promotion concurrente l'" Antiquité tardive ", qui veut traduire l'idée que les sociétés du III ${ }^{\mathrm{e}}$ au VI $\mathrm{VI}^{\mathrm{e}}$ siècle ap. J.-C. (voire après) aient été capables d'intégrer le principe de changement à une conception de l'identité comme héritage d'une tradition et continuité ou réactualisation du même. Sur les sociétés "froides " ou " chaudes ", cf. C. LÉVI-STRAUSS, "Le champ de l'anthropologie" (1960), repris in Anthropologie structurale deux, Paris, Plon, 1973, p. 39-42 ; F. HARTOG, Régimes d'historicité. Présentisme et expériences du temps, Paris, Seuil, 2003, p. 34-38.

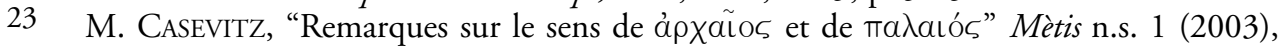
p. 125-136 ; cf. également ci-après les remarques de P. PAYEN, "Préhistoire de l'humanité et temps de la cité : l'"Archéologie » de Thucydide". 
lien concret avec le présent, à la manière du " present perfect " de la grammaire anglaise ; le second en est séparé et abstrait : un prétérit. Une " archéologie ", récit des choses anciennes, présente un passé continu et bien lisible. À l'occasion, elle n'en sait pas moins exhumer une antiquité morte pour méditer sur son étrange altérité. Entre distance et interférence, l'expérience du temps se prête à de tels courts-circuits.

La Préhistoire des Anciens est ainsi entendue, au sens d'un discours sur le passé antérieur, au sens d'une archéologie, voire d'une "paléologie " du politique. Elle est lisible, dans l'historiographie, l'étiologie, la tradition philosophique et la littérature ethnographique, comme un modèle théorique, altérité indéchiffrable ou objet inconcevable. Au cours de la journée d'études organisée par ERASME, la réflexion s'est organisée autour de quelques interrogations : dans l'historiographie antique, le passé antérieur est-il connaissable ? comment la préhistoire est-elle mise en récit et quel lien entretient-elle avec l'intelligence ou la perception du présent ? À quelles conditions et de quelles façons le passé antérieur à la cité s'est-il perpétué dans la mémoire collective ? Comment le passage de l'état présocial à une société organisée est-il représenté ?

Les articles qui suivent abordent ces questions à partir de corpus étalés, pour l'essentiel, du IVe siècle av. au II siècle ap. J.-C., et constitués au sein de genres aussi distincts que l'historiographie de Thucydide, les dialogues platoniciens, la poésie philosophique de Lucrèce, les excursus ethnographiques des géographes et des naturalistes romains, et l'éloquence épidictique d'Aelius Aristide. Ces textes sont disjoints par leurs modes d'expression, leur régime d'élocution leurs usages culturels, mais aussi par leur point de vue et la couleur spécifique qu'ils donnent à la tradition dans laquelle ils prennent place. Mais ils dessinent les contours d'un espace de représentations partagées et d'une anthropologie dont la cité constitue le point focal : par-delà les interprétations individuelles et les variations contextuelles, ils ne conçoivent la "préhistoire " que difficilement, l'état social ne semblant susceptible de trouver de formulation positive que par référence à l'ordre du politique. Ils ne voient le passé antérieur qu'à la lumière de la cité, comme son brouillon, son repoussoir ou son envers symétrique, voire comme un passé rationnellement nécessaire à la généalogie du présent, mais impensable dans d'autres termes que ceux de la cité - avec ou contre elle.

Pascal PAYEN s'attache à l'" Archéologie » de Thucydide. La description des « temps anciens ", préhistoire de la guerre du Péloponnèse, a une fonction structurante dans l'œuvre de l'Athénien. Elle collecte une série de commencements qu'elle identifie et nomme. Bien que le récit dispose des repères temporels et oppose un temps jadis

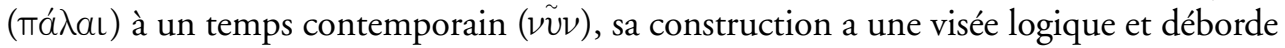
cette opposition. La relégation de l'ordre temporel à un plan secondaire développe le principe de l'universalité de la nature humaine et saisit les transformations des sociétés d'une manière synchronique. La préhistoire n'est saisissable qu'à travers des analogies et des inférences tirées du présent. Cette méthode caractérise une histoire conceptualisante dont le présent constitue le foyer. 
Marie-Laurence DesClOS examine la question du passé antérieur dans le Timée, le Critias et les Lois. Le récit de la " préhistoire " relève du mytheux et s'oppose à l'histoire comme savoir au moins vraisemblable, tissu de conjectures, de fictions et de reconsti-

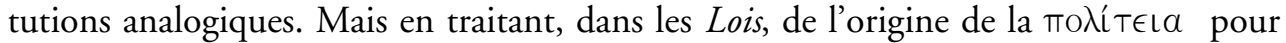
fonder les institutions de la cité des Magnètes, Platon élabore une séquence chronologique qui lui permet de construire un modèle argumentatif : une fois les premières origines effacées et rendues illisibles, il peut retracer la naissance de la société humaine en décomposant par raisonnement le passage du multiple à l'un et du naturel au conventionnel, pour aboutir à une doctrine du contrat social.

José KanY-TurPIn, dans le Chant $\mathrm{V}$ du De rerum natura, repère la vision composite d'une " préhistoire » définie comme la geste de l'humanité avant l'invention de l'écriture. Fidèle à la recherche épicurienne des uestigia, le raisonnement sur les indices doit permettre de dégager le modèle d'évolution d'un monde sans providence, sans finalité ni principe extérieur de changement, et de retracer le passage de l'état de violence universelle à l'époque où, de guerre lasse, l'humanité souscrivit un pacte de droit et se plia à la justice. Lucrèce historicise ce passage pour rejeter la définition de l'homme comme animal politique, mais aussi pour réintégrer les formes les plus anomiques de la violence au présent, en contemporain des derniers soubresauts de la République romaine.

Pierre CORDIER considère la représentation des primitifs contemporains chez les ethnographes romains. Il s'agit là d'une forme d'historicisation, dans la mesure où le présent de ces groupes sociaux appelle constamment des analogies avec le passé des sociétés du cultus. L'espace romain voit coexister des époques techniques différentes, que la diffusion des façons de faire romaines fait tendre vers l'homogénéité. L'ethnographie alimente ainsi une histoire de la culture, qui n'est pas dépourvue de perspectives téléologiques, et montre à l'œuvre un processus de civilisation intrinsèquement lié à la cité romaine.

Estelle OUdoT examine les premiers temps d'Athènes selon le Panathénaïque d'Aelius Aristide. Il ne conteste pas l'Empire romain, dont il entreprend de concilier et d'articuler la domination avec la suprématie athénienne. En remodelant l'«Archéologie » de Thucydide et les développements sur l'Athènes primite du Timée et du Critias, Aristide représente une cité apte à modéliser la forme parfaite d'exercice du pouvoir et à la situer en dehors de l'histoire. C'est le rôle de Rome que d'intégrer les valeurs grecques à l'histoire, révélant ainsi la place d'Athènes dans le processus de civilisation.

PIERRE CORDIER

Université de Toulouse II-Le Mirail

UFR d'Histoire, Arts et Archéologie

5, allées Antonio Machado

31058 Toulouse Cedex 9

pierre.cordier@univ-tlse2.fr 